Revista de Psicología de la PUCP. Vol. XX, 1, 2002

\title{
Adaptación del Inventario de Estilos de Personalidad de Millon en una población universitaria peruana ${ }^{1}$
}

\author{
María del Pilar Sánchez-López ${ }^{2}$ \\ Universidad Complutense de Madrid \\ Cecilia Thorne ${ }^{3}$, Patricia Martínez ${ }^{4}$, Isabel Niño de Guzmán ${ }^{5}$ Doris Argumedo ${ }^{6}$ \\ Pontificia Universidad Católica del Perú
}

\begin{abstract}
El objetivo del estudio fue la adaptación del MIPS (1994) a una población de 390 estudiantes universitarios de Lima (188 hombres y 202 mujeres). Se adaptó la prueba al castellano hablado en el Perú. El análisis psicométrico mostró un índice de confiabilidad promedio de 70 y una validez interna estructural. La mayoría de las escalas presentó niveles de consistencia interna aceptables. La comparación con los estudios realizados en España y EEUU mostró que la confiabilidad promedio es semejante a la hallada en la población española y ligeramente inferior a la norteamericana. El análisis de las diferencias entre los promedios en cada una de las escalas indicó mayores divergencias entre la población peruana y la española que entre la población peruana y la norteamericana.

Palabras clave: Inventario de Personalidad de Millon, MIPS, adaptación de pruebas, personalidad, universitarios, Perú.
\end{abstract}

The adaptation of the Millon Index of Personality Styles to a Peruvian population of university students

The purpose of the study was the adaptation of the MIPS (1994) to a population of 390 university students of Lima (188 men and 202 women). The inventory was adapted to the Spanish spoken in Peru. The psychometrical analysis revealed a reliability index of .70 as well as a structural internal validity. Most of the scales presented acceptable levels of internal consistency. The comparison with the studies carried out in Spain and USA showed that the levels of internal-consistency were similar to those found in the Spanish population and slightly below to those found in the North American population. The analysis of the differences between the averages in each one of the scales indicated greater discrepancies between the Peruvian population and the Spanish, than between the Peruvian population and the North American.

Key words: Millon Index of Personality Styles, MIPS, test adaptation, personality, university students, Peru.

1 Esta investigación fue financiada por la Dirección Académica de Investigación de la PUCP. En la fase de la adaptación y la administración de las pruebas se contó con el apoyo de Yojana Pavón, alumna de psicología de la Universidad Complutense de Madrid y de Carla Albornoz, Nélly Huapaya, Brígida Mata, Rosario Paredes, Mónica Suyo, Roxana Velásquez, Carla Vidal e Ivette Villanueva, alumnas de la especialidad de psicología de la PUCP.

2 Doctora en Psicología. Catedrática de Psicología Diferencial y directora del Departamento de Psicología Diferencial y Psicología del Trabajo. Es autora de diversos artículos en 

El uso de inventarios de personalidad es una práctica común entre los psicólogos peruanos para llevar a cabo evaluaciones clínicas o de selección de personal. Sin embargo, se siguen utilizando versiones de instrumentos psicológicos que no se encuentran adaptados a la realidad, resultando escasos los estudios de estandarización y baremación de pruebas psicológicas. El uso de pruebas no adaptadas suscita una serie de dificultades en la interpretación de resultados, produciéndose muchas veces sesgos negativos, dado que se trata de materiales creados dentro de contextos culturales diferentes. Por lo tanto, no basta contar con los instrumentos en castellano, sino que también deben ser adaptados y probados en una población con el objeto de determinar su validez y confiabilidad.

En una investigación realizada sobre la evaluación psicológica en el Perú (Thorne, 2001) se percibe que en los últimos años se han incrementado las investigaciones en este campo, comparado con un estudio previo (Thorne, Escurra y Delgado, 1993). Asimismo, se apre-

revistas españolas e internacionales y ha publicado ocho libros. Su línea de investigación comprende los estilos de personalidad, el bilingüismo, la cronopsicología, el rendimiento y la salud. Correo electrónico: psdif02@sis.ucm.es.

3 Doctora en Psicología. Profesora principal y Directora Académica de Planeamiento y Evaluación. Sus trabajos de investigación comprenden el desarrollo del niño en ambientes desfavorecidos, la evaluación psicológica y el aprendizaje y la enseñanza de la lectura, temas sobre los cuales ha publicado numerosos artículos en revistas nacionales e internacionales, capítulos de libros y libros. Correo electrónico: cthome@pucp.edu.pe.

4 Magíster en Psicología. Profesora asociada. Coordinadora de la Sección Psicología y de la Maestría en Estudios Teóricos en Psicoanálisis. Candidata al doctorado en la Universidad Autónoma de Barcelona. Especialista en estudios de evaluación y diagnóstico de adolescentes, tema sobre el cual ha realizado varias investigaciones y publicado artículos. Correo electrónico: pmartin@pucp.edu.pe.

5 Magíster en Psicología. Profesora auxiliar. Especialista en evaluación y uso de pruebas psicológicas. Ha realizado investigaciones y publicado artículos sobre estos temas. Correo electrónico: inino@pucp.edu.pe.

6 Licenciada en Psicología Clínica. Profesora de Psicología. Especialista en metodología de investigación y psicometría. Está realizando una maestría en Estudios Teóricos en Psicoanálisis. Correo electrónico: dargume@pucp.edu.pe 
cia que hay un esfuerzo por adaptar pruebas y crear baremos, así como por elaborar instrumentos para la realidad peruana. Lamentablemente la difusión de los estudios, a través de publicaciones, no necesariamente es utilizada por los profesionales en su trabajo diario profesional. El estudio pone de manifiesto, asimismo, que muchos profesionales desconocen las investigaciones realizadas, apreciándose una marcada tendencia a usar pruebas desarrolladas y publicadas en otros países de habla hispana o pseudoadaptaciones realizadas por ellos mismos.

El Inventario de Estilos de Personalidad de Millon (MIPS) fue elaborado por Theodore Millon en 1994 para evaluar la personalidad desde una perspectiva de normalidad y no de patología. La prueba busca medir toda la gama del funcionamiento normal y se basa en el modelo de personalidad desarrollado por Millon. Se trata de un modelo que es integrador en la medida que propone una serie de estilos y prototipos que son esencialmente nomotéticos, al cual incorpora el concepto de polaridad. Añade, asimismo, un rango de subtipos de personalidad que tiende a ser un estudio más específico o idiográfico.

El instrumento resulta de particular interés dado que su estructura interna da cuenta del esfuerzo por operativizar una concepción teórica de la personalidad desde una perspectiva dinámica. En este sentido, el modelo propuesto por Millon representa a la personalidad como un conjunto de disposiciones en interacción dinámica que, al combinarse, dan origen a las diversas configuraciones denominadas estilos de personalidad.

El modelo está representado en la prueba por 24 escalas de personalidad agrupadas en 12 pares, cada una de las cuales incluye dos escalas yuxtapuestas que simbolizan las polaridades. Estas se encuentran distribuidas en tres grandes áreas: metas motivacionales, modos cognitivos y conductas interpersonales. Asimismo, contiene tres índices de control que son la impresión positiva, la impresión negativa y la consistencia que se refiere al índice de control de la sinceridad. En el 
Cuadro 1 aparecen las escalas del MIPS. El lector puede remitirse al manual de la prueba para una descripción detallada de cada una de las escalas. En castellano hay dos versiones del manual del MIPS, una argentina realizada por Casullo en 1997 y otra española realizada por Sánchez López, Díaz Morales y Aparicio García en el 2001.

\section{Cuadro 1}

Las escalas del MIPS

\begin{tabular}{|llll|}
\hline $\begin{array}{c}\text { Metas } \\
\text { motivacionales }\end{array}$ & $\begin{array}{c}\text { Modos } \\
\text { cognitivos }\end{array}$ & $\begin{array}{c}\text { Conductas } \\
\text { interpersonales }\end{array}$ & $\begin{array}{c}\text { Índices de } \\
\text { control }\end{array}$ \\
\hline $\begin{array}{l}\text { Apertura } \\
\text { Preservación }\end{array}$ & $\begin{array}{l}\text { Extraversión } \\
\text { Introversión }\end{array}$ & $\begin{array}{l}\text { Retraimiento } \\
\text { Comunicatividad }\end{array}$ & Impresión positiva \\
Modificación & Sensación & Vacilación & Impresión negativa \\
Acomodación & Intuición & Firmeza & \\
Individualismo & Reflexión & Discrepancia & Consistencia \\
Protección & Afectividad & Conformismo & \\
& Sistematización & Sometimiento & \\
& Innovación & Control & \\
& & Insatisfacción & \\
& & Concordancia & \\
\hline
\end{tabular}

El cuestionario está dirigido a personas con educación secundaria y de más de 18 años. Está compuesto por 180 ítems con afirmaciones de verdadero o falso que pueden ser respondidas en aproximadamente 30 minutos.

El presente estudio ha tenido como objetivo la adaptación del MIPS a una población de estudiantes universitarios. Se inserta dentro del conjunto de investigaciones realizadas con el propósito de aportar a la adaptación del instrumento al castellano y a la recolección de evidencia empírica que pueda sustentar la validez y confiabilidad del mismo en diversos contextos culturales (Sánchez López y Casullo, 2000).

Los objetivos del presente estudio fueron:

- Adaptar el contenido del Inventario de Estilos de Personalidad de Millon al castellano hablado en el Perú. 
- Estudiar la confiabilidad y la validez del instrumento aportando a la contrastación empírica del mismo en un nuevo contexto cultural.

- Desarrollar un estudio transcultural comparando los resultados peruanos con los hallados en investigaciones anteriores en España y la muestra estadounidense con la que se validó originalmente el inventario.

\section{Metodología}

\section{Participantes}

Los participantes fueron un grupo de estudiantes provenientes de tres facultades de una universidad privada de Lima (Estudios Generales Letras, Letras y Ciencias Humanas, y Ciencias e Ingeniería). Se realizó un muestreo al azar dentro de las facultades a las cuales se les aplicaría la prueba. El grupo final estuvo conformado por 390 estudiantes universitarios, de los cuales 188 eran hombres (48.2\%) y 202 mujeres (51.8\%). La edad de los estudiantes oscilaba entre los 16 y los 40 años, siendo el promedio de 20 años. La mayoría (65.6\%) se encontraban entre los 16 y 20 años de edad tal como se describe en el Cuadro 2, donde aparece la distribución de los participantes por edad y sexo.

\section{Cuadro 2}

Distribución de los estudiantes por edad y sexo

\begin{tabular}{|cccc|}
\hline Edad & $\begin{array}{c}\text { Hombres } \\
\text { Universitarios }\end{array}$ & $\begin{array}{c}\text { Mujeres } \\
\text { Universitarias }\end{array}$ & Total \\
\hline $16-20$ & 111 & 143 & 254 \\
$21-25$ & 68 & 52 & 120 \\
26 a 40 & 9 & 7 & 16 \\
\hline Total & 188 & 202 & 390 \\
\hline
\end{tabular}

\section{Instrumentos}

El instrumento utilizado fue el Inventario de Estilos de Personalidad de Millon - adaptación peruana. Para poder realizar la adecuación 
linguística del MIPS al castellano hablado en el Perú se llevó a cabo una revisión exhaustiva del contenido del inventario en inglés (Millon, 1994) y sus adaptaciones al castellano para la población argentina (Millon, 1997) y española (Millon, 2001). Cuatro profesionales familiarizados con la teoría que sostiene la estructura y el contenido del instrumento participaron en la elaboración de la primera versión que sirvió de piloto. Esta versión fue aplicada a un grupo de diez estudiantes universitarios, solicitándoseles que anotasen aquellos ítems poco claros o frente a los cuales tuvieran dudas respecto a su significado. Aquellos ítems que presentaron dificultades fueron analizados nuevamente por el grupo de expertos y rediseñados de modo que no hubiese discrepancias respecto a sus significados hasta alcanzar un consenso entre los miembros del equipo de investigación. En el Cuadro 3 se muestran algunos ejemplos de la adecuación lingüística y de las semejanzas y diferencias entre las tres versiones en castellano.

\section{Cuadro 3}

Algunos ejemplos de la adecuación lingüística de los items

\begin{tabular}{|c|c|c|c|}
\hline Ítem & Versión argentina & Versión española & Versión peruana \\
\hline $2^{a}$ & $\begin{array}{l}\text { Siempre hice lo que } \\
\text { quise y asumí las con- } \\
\text { secuencias. }\end{array}$ & $\begin{array}{l}\text { Siempre he hecho las co- } \\
\text { sas a mi manera y he acep- } \\
\text { tado las consecuencias. }\end{array}$ & $\begin{array}{l}\text { Siempre he hecho lo que } \\
\text { he querido y he acepta- } \\
\text { do las consecuencias. }\end{array}$ \\
\hline $3^{h}$ & $\begin{array}{l}\text { Me gusta hacerme car- } \\
\text { go de una tarea. }\end{array}$ & $\begin{array}{l}\text { Me gusta ser la persona } \\
\text { que asume el control de } \\
\text { las cosas. }\end{array}$ & $\begin{array}{l}\text { Me gusta ser la perso- } \\
\text { na que asume el con- } \\
\text { trol de las cosas. }\end{array}$ \\
\hline 6 & $\begin{array}{l}\text { A veces me las arreglo } \\
\text { para arruinar las cosas } \\
\text { buenas que me pasan. }\end{array}$ & $\begin{array}{l}\text { A veces me las arreglo } \\
\text { para estropear las cosas } \\
\text { buenas que me pasan. }\end{array}$ & $\begin{array}{l}\text { A veces me las arreglo } \\
\text { para arruinar las cosas } \\
\text { buenas que me pasan. }\end{array}$ \\
\hline $8^{d}$ & $\begin{array}{l}\text { Preferiría ser un segui- } \\
\text { dor más que un líder. }\end{array}$ & $\begin{array}{l}\text { Preferiría ser un segui- } \\
\text { dor más que un líder. }\end{array}$ & $\begin{array}{l}\text { Preferiría ser un segui- } \\
\text { dor más que un líder. }\end{array}$ \\
\hline
\end{tabular}

Nota.

a En las tres versiones el ítem es diferente

b En la versión española y la peruana el item es igual.

- En la versión argentina y la peruana el ítem es igual.

* En las tres versiones el ítem es igual. 
La versión final peruana de la prueba tal como fue aplicada a los participantes del estudio aparece en el Anexo A.

El procedimiento para administrar las pruebas se hizo a través de la aplicación grupal directa o in situ del inventario durante los horarios de clase de los estudiantes en sus respectivas facultades. Previamente se solicitó autorización al profesor del curso, del mismo modo la participación de los estudiantes fue voluntaria.

\section{Resultados y discusión}

Los resultados se han dividido de acuerdo a los objetivos del estudio en dos partes. En la primera parte se presentan los análisis psicométricos que permiten realizar los estudios de confiabilidad y validez. En la segunda parte se realiza la comparación transcultural de la muestra universitaria peruana con la muestra norteamericana y española.

\section{Análisis psicométrico del MIPS}

Se calcularon los índices de tendencia central y dispersión para cada una de las escalas del instrumento, así como las distribuciones de frecuencias e histogramas para determinar descriptivamente el nivel de simetría de las mismas. Igualmente, se realizó la prueba de Kolmogorov-Smirnoff para establecer la normalidad en la distribución de las puntuaciones en cada escala. Las puntuaciones de prevalencia para el Perú aparecen en el Manual Español publicado por TEA en el 2001 (Millon), donde también aparecen las puntaciones para España, Argentina y México.

En general, las medidas de tendencia central son cercanas a las encontradas en la muestra universitaria española y la estadounidense. Posteriormente, se analizan con mayor detalle las diferencias de promedios encontradas entre las poblaciones. 
Por otro lado, la mayoría de las escalas (15 de 24) muestran una distribución normal en sus puntuaciones, observándose en las demás un ligero sesgo acorde con la significación teórica de las escalas: en las escalas con una connotación positiva aparece una ligera asimetría negativa, mientras que en las escalas que hacen referencia a constructos negativos la asimetría es positiva.

Para el cálculo de la confiabilidad, se llevaron a cabo los análisis de consistencia interna entre los ítems de cada escala, determinando la proporción de la varianza de la puntuación total de la escala que es atribuible a la fuente de variación común de los ítems, es decir a las puntuaciones verdaderas del constructo medido (Aiken, 1996). Se utilizó el coeficiente alfa de Cronbach para estudiar la confiabilidad por consistencia interna, considerando la ponderación de los prototípicos y complementarios establecidas por Millon para la versión estadounidense. Posteriormente, se realizó un segundo análisis de confiabilidad aplicando el coeficiente Kuder-Richarson, un caso especial del coeficiente alfa para ítems dicotómicos (Aiken).

Los índices de confiabilidad, tomando en cuenta la ponderación de los ítems prototípicos y complementarios, oscilan entre .59 y .84 con un promedio de .71 (.72 para la muestra española y .77 para la norteamericana). Las escalas más confiables pertenecen a las áreas de Conductas Interpersonales (Vacilación: 84 y Firmeza: .79) y Metas Motivacionales (Preservación: .80). El área de Modos Cognitivos contiene las escalas con confiabilidades más bajas tales como Intuición con .59 , Sensación e Innovación cada cual con un alfa de .60 (ver Anexo B).

Los niveles de consistencia interna logrados por las escalas, estableciendo pesos similares para todos los ítems a partir de su dicotomización, son semejantes a los encontrados en los análisis de confiabilidad que incluyen la ponderación de ítems (ver Anexo B). 
En relación con la validez del instrumento se evaluó la estructura interna del MIPS analizando las intercorrelaciones entre las escalas para contrastar empíricamente las convergencias o divergencias entre las mismas y su congruencia con el modelo teórico que las sustenta. Las intercorrelaciones entre las escalas se desarrollaron considerando tanto la totalidad de los ítems que componen cada escala, como utilizando sólo los ítems prototípicos como un medio de control de la influencia de la superposición de los ítems complementarios en la covariación de las escalas (Millon, 1997).

La validez interna de un instrumento se relaciona con la pauta de convergencia y divergencia entre sus escalas. En este sentido, las escalas que miden constructos afines deberían converger y las que miden constructos inconexos deberían divergir (Millon).

Como era de esperar, teniendo en cuenta la teoría y los procedimientos de construcción, se pudo constatar que las escalas polares opuestas presentan correlaciones negativas elevadas. También de acuerdo a lo previsto, se observaron correlaciones altas y positivas entre constructos teóricamente asociados, tales como por ejemplo: Vacilación (9A) y Preservación (1B),$\quad r=.811$; Firmeza (9B) y Comunicatividad $(8 \mathrm{~B}), r=.758$. Del mismo modo, los resultados muestran correlaciones negativas elevadas entre constructos teóricamente discordantes, tal como por ejemplo: Retraimiento (8A) y Extraversión (4A), $r=-.792$; Concordancia (12B) y Control (11B), $r=$ -.704). Por último se hallaron correlaciones nulas próximas a cero entre constructos teóricamente no asociados como: Individualismo (3A) y Preservación (1B), $r=.004$; Control (11B) y Retraimiento (8A), $r=-.007$ (ver Anexo C).

Si bien, la medida en que los ítems del MIPS se superponen refuerza significativamente la magnitud de las intercorrelaciones entre las escalas (Millon, 1997), se pudo observar que estas asociaciones se sustentan ante todo en las relaciones fenomenológicas de estos 
constructos en la realidad. Así, el patrón general de relaciones convergentes y divergentes entre las escalas del MIPS que se obtuvo utilizando sólo los ítems prototípicos es muy semejante al encontrado utilizando la totalidad de los ítems.

\section{Comparación transcultural de la muestra universitaria peruana con la muestra norteamericana y española}

Se calcularon las diferencias entre los promedios en cada una de las escalas del MIPS, entre la muestra universitaria peruana y la muestra norteamericana y española, contrastando la significación poblacional de estas diferencias a través de la prueba $t$ de Student. Se incluyó además el análisis del tamaño del efecto siguiendo las recomendaciones de Cohen (1988) como una medida de la potencia estadística. El tamaño del efecto es definido, en el contexto de una prueba de significación, como el grado en que la hipótesis nula es falsa (Cohen). El tamaño del efecto responde, en este caso, a la pregunta sobre la cantidad en que se manifiesta una diferencia estadísticamente significativa (Sánchez López y Casullo, 2000).

Se tomaron en cuenta las diferencias de promedios con un nivel de significación $p<.0001$ y con un tamaño del efecto considerable $(d>.3)$, moderado $(d>.5)$ y amplio $(d>.8)$.

Considerando la muestra peruana y norteamericana, encontramos diferencias significativas con tamaños del efecto moderados en las áreas de Modos Cognitivos y Relaciones Interpersonales (ver Anexo D). Estos resultados se grafican en la Figura 1. 


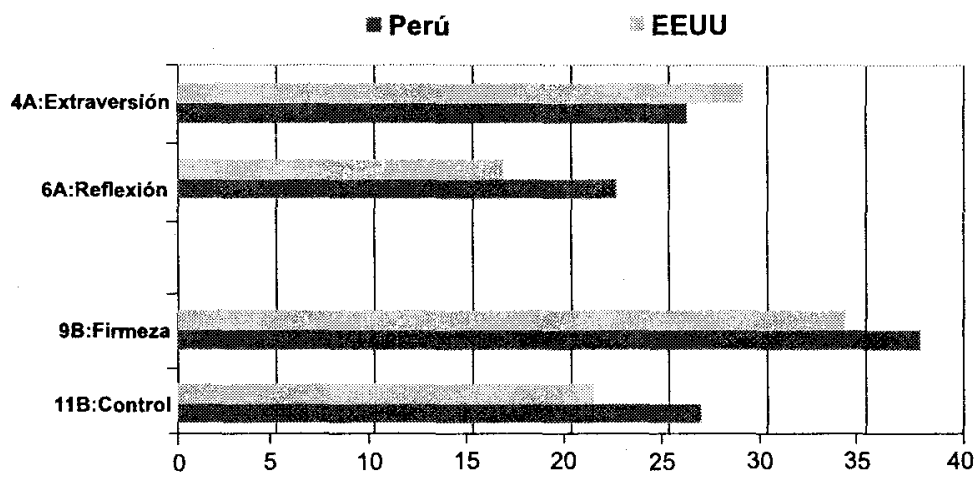

Figura 1. Diferencia entre estudiantes peruanos y estudiantes norteamericanos.

Tal como se puede apreciar en la Figura 1, los universitarios norteamericanos tienden a ser más extravertidos y muestran una mayor tendencia a apoyarse en los demás como fuente de estimulación y aliento, mientras que los peruanos están más orientados a acercarse al mundo y procesar los conocimientos por medio de la lógica y el razonamiento analítico, basando sus decisiones en juicios impersonales y objetivos y no en emociones subjetivas. En el área Relaciones Interpersonales, observamos discrepancias en las escalas de Firmeza y Control. Los peruanos tienden a percibirse como más competentes y talentosos que quienes los rodean, manifestando seguridad y confianza en sí mismos (Firmeza) así como un mayor autodominio, alta fuerza del yo y control sobre los demás en su estilo de relación interpersonal (Control).

Las diferencias entre universitarios peruanos y españoles son mayores que las observadas en relación con la población norteamericana tal como se aprecia en la Figura 2. 


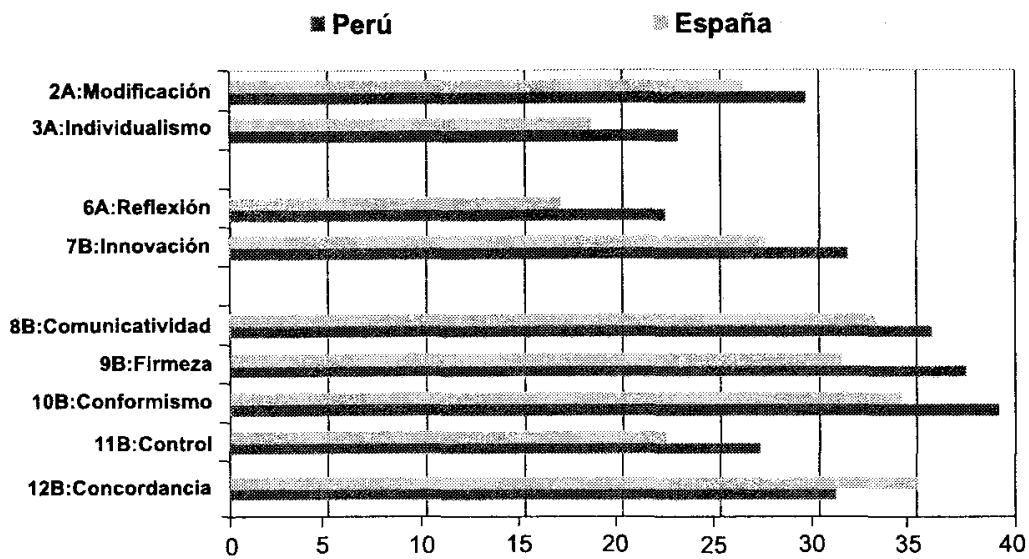

Figura 2. Diferencias entre universitarios peruanos y españoles.

Hallamos así diferencias con tamaño de efecto moderados en las tres áreas que componen el inventario. En el área de Metas Motivacionales, encontramos que los estudiantes peruanos están más orientados a modificar activamente su entorno y los sucesos de su vida (Modificación), siendo menos proteccionistas y preocupándose menos por las necesidades de los demás (Individualismo) que sus pares españoles. En el área de los Modos Cognitivos, aparecen como más reflexivos, basando sus decisiones en juicios objetivos más que en emociones (Reflexión), estando más prontos a modificar y reordenar creativamente cualquier cosa que signifique un obstáculo y transformar lo dado produciendo consecuencias nuevas e imprevistas (Innovación). Como estilo de relación interpersonal, los peruanos tienden a buscar mayor estimulación, excitación y atención (Comunicatividad), se muestran más confiados y seguros de sí mismos (Firmeza), están más dispuestos a seguir pautas y normas sociales (Conformismo), manifiestan un mayor control y autodominio en la relación con los demás (Control), tendiendo a establecer vínculos afectivos y lealtades muy fuertes (Concordancia). Para mayores detalles ver el Anexo E. 


\section{Comentarios finales}

Si bien existen versiones en castellano del Inventario de Estilos de Personalidad de Millon, las investigaciones realizadas en Argentina, España y Perú demuestran que es necesario adaptar el contenido del mismo al castellano particular de cada país. En este sentido, encontramos que la adaptación lingüística realizada cumple con los requisitos necesarios para ser utilizada en población universitaria peruana.

En cuanto a la confiabilidad promedio del MIPS encontramos que ésta es semejante a la hallada en la población española y ligeramente inferior a la registrada por Millon en población norteamericana. Evaluando las escalas de modo particular, la mayoría presenta niveles de consistencia interna aceptables; sin embargo, es necesario evaluar con mayor profundidad el comportamiento del área Modos Cognitivos pues contiene las escalas con las más bajas confiabilidades.

El instrumento presenta validez interna estructural, lo cual demuestra que las escalas del MIPS están diseñadas para representar plenamente un conjunto de estilos de personalidad concordantes con la teoría, multidimensionales e intercorrelacionados.

La comparación con las muestras norteamericana y española indica que existen mayores diferencias con los universitarios españoles. Resulta de interés establecer comparaciones con poblaciones con contextos culturales más cercanos como la argentina, en donde ya se han desarrollado estudios de adaptación y estandarización del MIPS. Asimismo, cabe señalar que los participantes de este estudio provienen de una sola universidad, que tiene características propias y que no necesariamente es representativa de una población universitaria peruana típica. 
Se requiere ampliar la investigación sobre la prueba y profundizar en estudios sobre su comportamiento psicométrico en población adulta y estratos socioeconómicos y culturales distintos, de modo que se pueda contar con un instrumento válido y confiable que pueda ser aplicado a la población peruana en general.

\section{Referencias}

Aiken, L. (1996). Tests psicológicos y evaluación. México: Prentice Hall International.

Casullo, M. (1997). Cuadernillo de administración del MIPS. Buenos Aires: Paidós.

Cohen, J. (1988). Statistical power analysis for the behavioral sciences. Nueva Jersey: Erlbaum.

Millon, T. (1994). Millon Index of Personality Styles. San Antonio: The Psychological Corporation.

Millon, T. (1997). Inventario Millon de Estilos de Personalidad. Buenos Aires: Paidós.

Millon, T. (2001). Inventario de Estilos de Personalidad de Millon. Adaptación Española: Sánchez López, M. P., Díaz Morales, J. F., Aparicio García, M. Madrid: TEA.

Sánchez López, M. y Casullo, M. (2000). Los estilos de personalidad: una perspectiva iboeroamericana. Madrid: Miño y Dávila.

Thorne, C. (2001). La evaluación psicológica en la formación, el ejercicio profesional y la investigación en Lima. Conferencia Magistral presentada en el X Congreso Nacional de Psicología. Lima, 27-29 de setiembre.

Thorne, C., Escurra, M. y, Delgado, A. (1993). Investigaciones sobre pruebas psicológicas en el Perú. Ponencia presentada en el XXIV Congreso Interamericano de Psicología, Santiago de Chile, $4-9$ de julio. 


\section{Anexo A \\ Inventario Millon de Estilos de Personalidad \\ Theodore Millon \\ Adaptación Peruana}

\section{Instrucciones}

Por favor, lea cuidadosamente estas instrucciones antes de empezar. Luego marque todas sus contestaciones en la Hoja de respuestas.

Este cuestionario contiene una lista de frases. Lea cada frase con atención y piense si lo que dice describe o no su forma de ser. Si usted está de acuerdo con ella, marque Su respuesta en V (verdadero). Si usted está en desacuerdo, piensa que no describe su forma de ser, marque F (falso) en la Hoja de respuestas.

Vea los ejemplos E1 y E2 que vienen aquí debajo y cómo se ha contestado a la derecha.

E 1.- Me gustaría pilotear una nave espacial

E2.- A la hora de vestirme prefiero los tonos oscuros

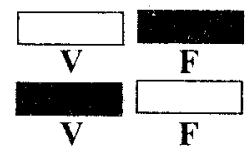

La persona que ha contestado a estos ejemplos ha indicado que es falso (F) que a ella le guste pilotear una nave espacial; pero es verdadero (V) que prefiere los tonos oscuros a la hora de vestirse. Como verá, no hay respuestas "correctas" ni "incorrectas"; por tanto, trate de ser lo más sincero posible.

Anote una respuesta a todas las frases y asegúrese de que marca cada respuesta en la línea correspondiente a la misma frase y en la opción ( $\mathrm{V} \circ \mathrm{F}$ ) que mejor se ajusta a su manera de ser. Intente decidirse siempre por una de estas dos opciones, procurando no dejar ninguna respuesta sin contestar.

ESPERE, NO PASE LA PAGINA HASTA QUE SE LO INDIQUEN. 
1. Soy una persona tranquila y colaboradora.

2. Siempre he hecho lo que he querido y he aceptado las consecuencias.

3. Me gusta ser la persona que asume el control de las cosas.

4. Tengo una manera habitual de hacer las cosas, con lo que evito equivocarme.

5. Contesto las cartas el mismo día que las recibo.

6. A veces me las arreglo para arruinar las cosas buenas que me pasan.

7. Ya no me entusiasman tantas cosas como antes.

8. Preferiría ser un seguidor más que un líder.

9. Me esfuerzo por llegar a ser popular.

10. Siempre he tenido talento para tener éxito en lo que hago.

11. Con frecuencia me doy cuenta de que he sido tratado injustamente.

12. Me siento incómodo cuando me tratan bien.

13. Con frecuencia me siento tenso en situaciones sociales.

14. Creo que la policía abusa del poder que tiene.

15. Algunas veces he tenido que ser bastante brusco con la gente.

16. Los niños deben obedecer siempre las indicaciones de sus mayores

17. A menudo me molestan la forma en que se hacen las cosas.

18. A menudo espero que me pase lo peor.

19. No me importaría tener pocos amigos.

20. Soy tímido e inhibido en situaciones sociales.

21. Aunque esté en desacuerdo, por lo general dejo que la gente haga lo que quiera.

22. Es imposible pretender que las personas digan siempre la verdad.

23. Puedo hacer comentarios desagradables si considero que las personas se los merecen.

24. Me gusta cumplir con lo establecido y hacer lo que se espera de mí.

25. Muy poce de lo que hago es valorado por los demás.

26. Casi todo lo que intento hacer me resulta fácil.

27. Últimamente me he convertido en una persona más encerrada en mí misma. 
28. Tiendo a hacer un drama de las cosas que me pasan.

29. Siempre trato de hacer lo que es correcto.

30. Dependo poco de la amistad de los demás.

31. Nunca he estado estacionado durante más tiempo del que un parquímetro establecía como límite.

32. Los castigos nunca me han impedido hacer to que he querido.

33. Me gusta organizar todas las cosas hasta en sus mínimos detalles.

34. A menudo los demás logran irritarme.

35. Jamás he desobedecido las indicaciones de mis padres.

36. Siempre logro conseguir lo que quiero aunque tenga que presionar a los demás.

37. Nada es más importante que proteger la reputación personal.

38. Los demás tienen mejores oportunidades que yo.

39. Ya no expreso lo que realmente siento.

40. Es imposible que lo que tengo que decir interese a los demás.

41. Me esfuerzo por conocer gente interesante y tener aventuras.

42. Me tomo con poca seriedad muchas de las responsabilidades que tengo.

43. Soy una persona dura, nada sentimental.

44. Pocas cosas en la vida pueden conmoverme.

45. Me pone muy nervioso el tener que conocer y conversar con gente nueva.

46. Soy una persona colaboradora que cede ante los demás.

47. Actúo en función del momento, dependiendo de las circunstancias.

48. Primero planifico y luego sigo activamente el plan trazado.

49. A menudo me he sentido inquieto con ganas de irme a cualquier parte.

50. Lo mejor es controlar nuestras emociones

51. Desearía que la gente no me culpara a mí cuando algo sale mal.

52. Probablemente soy mi peor enemigo.

53. Tengo muy pocos lazos afectivos fuertes con otras personas.

54. Me siento intranquilo con personas que no conozco muy bien.

55. Es correcto tratar de burlar la ley sin dejar de cumplirla. 
56. Hago mucho por los demás, pero ellos hacen poco por mí.

57. Siempre he creído que los demás no tienen buena opinión de mí.

58. Tengo mucha confianza en mí mismo.

59. Sistemáticamente ordeno mis papeles y materiales de trabajo.

60. Mi experiencia me ha enseñado que las cosas buenas duran poco.

61. Algunos dicen que me gusta hacerme la víctima.

62. Me siento mejor cuando estoy solo.

63. Me pongo más tenso que los demás frente a situaciones nuevas.

64. Siempre trato de evitar las discusiones, por más que esté convencido de tener razón.

65. Busco situaciones novedosas y excitantes para mí.

66. Hubo épocas en que mis padres tuvieron problemas por mi comportamiento.

67. Siempre termino mi trabajo antes de descansar.

68. Otros consiguen cosas que yo no logro.

69. A veces siento que merezco ser infeliz.

70. Espero que las cosas sigan su curso antes de decidir qué hacer.

71. Procuro ocuparme más de los demás que de mí mismo.

72. A menudo creo que mi vida va de mal en peor.

73. El solo hecho de estar con otras personas me hace sentir inspirado.

74. Cuando manejo siempre estoy atento a las señales sobre límites de velocidad y procuro no excederme.

75. Uso mi cabeza y no mi corazón para tomar decisiones.

76. Generalmente suelo guiarme de mis intuiciones más que por la información que tengo sobre algo.

77. Jamás envidio los éxitos de los demás.

78. En el colegio me gustaban más los cursos prácticos que los teóricos.

79. Planifico las cosas con anticipación y actúo enérgicamente para que mis planes se cumplan.

80. Mi corazón controla mi cerebro.

81. Siempre puedo ver el lado positivo de la vida.

82. A menudo espero que alguien solucione mis problemas. 
83. Hago lo que quiero, sin pensar cómo va a afectar a los otros.

84. Reacciono con rapidez ante cualquier situación que pueda llegar a ser un problema para mí.

85. Sólo me siento una buena persona cuando ayudo a los demás.

86. Si algo sale mal, aunque no sea importante, se me arruina todo el día.

87. Disfruto más de mis fantasías que de la realidad cotidiana.

88. Me siento satisfecho dejando que las cosas ocurran.

89. Trato de ser más lógico que emocional.

90. Prefiero las cosas que se pueden ver y tocar antes que las que sólo se imaginan.

91. Me resulta difícil conversar con alguien que acabo de conocer.

92. Ser afectuoso es más importante que ser frío y calculador.

93. Las predicciones sobre el futuro son más interesantes para mí que los hechos del pasado.

94. Me resulta fácil disfrutar de las cosas

95. Me siento incapaz de influir en el mundo que me rodea.

96. Vivo según mis propias necesidades y no basado en las de los demás.

97. Nunca espero que las cosas pasen, hago que sucedan como yo quiero.

98. Evito contestar bruscamente cuando estoy molesto.

99. La necesidad de ayudar a otros guía mi vida.

100. A menudo me siento muy tenso, a la espera de que algo salga mal.

101. Ni siguiera siendo adolescente intenté copiar en un examen.

102. Siempre soy frío y objetivo en el trato con los demás.

103. Prefiero aprender a manejar un aparato antes que especular sobre por qué funciona de ese modo.

104. Soy una persona difícil de conocer bien.

105. Paso mucho tiempo pensando en los misterios de la vida.

106. Es fácil para mí controlar mis estados de ánimo.

107. Soy algo pasivo y lento en temas relacionados con la organización de mi vida. 
108. Hago lo que quiero sin importarme el complacer a otros.

109. Jamás haría algo malo, por más fuerte que sea la tentación de hacerlo.

110. Mis amigos y mis familiares recurren a mí en primer lugar para encontrar afecto y apoyo.

111. Aunque todo esté bien, generalmente pienso que va a pasar lo peor.

112. Planifico y organizo con cuidado mi trabajo antes de empezar a hacerlo.

113. Soy impersonal y objetivo al tratar de resolver un problema

114. Soy una persona realista a la que no le gustan las especulaciones.

115. Algunos de mis mejores amigos desconocen lo que realmente siento.

116. La gente piensa que soy una persona más racional que afectiva.

117. Mi sentido de la realidad es mejor que mi imaginación.

118. Primero me preocupo por mí y después de los demás.

119. Dedico mucho esfuerzo para que las cosas me salgan bien.

120. Siempre mantengo la compostura, sin importar lo que esté pasando.

121. Demuestro mucho afecto hacia mis amigos.

122. Muy pocas cosas me han salido bien.

123. Me gusta conocer gente nueva y saber cosas sobre sus vidas.

124. Puedo ignorar aspectos emocionales y efectivos en mi trabajo.

125. Prefiero ocuparme de realidades y no de posibilidades.

126. Necesito mucho tiempo para poder estar a solas con mis pensamientos.

127. Los sentimientos son más importantes que la lógica de la mente.

128. Me gustan más los soñadores que los realistas.

129. Soy más capaz que los demás de reírme de los problemas.

130. Es poco lo que puedo hacer, así que prefiero esperar a ver qué pasa.

131. Nunca me pongo a discutir, aunque esté muy enojado.

132. Expreso lo que pienso de manera franca y abierta.

133. Me preocupo por el trabajo que hay que realizar y no por lo que siente la gente que participa en su realización. 
134. Para mí lo ideal sería trabajar con ideas creativas.

135. Soy el tipo de persona que no se toma la vida muy en serio, prefiero ser más espectador que actor.

136. Me desagrada depender de alguien en mi trabajo.

137. Trato de asegurar que las cosas salgan como yo quiero.

138. Disfruto más de las realidades concretas que de las fantasías.

139. Muchas cosas sin importancia me ponen de mal humor.

140. A prendo mejor observando y hablando con la gente.

141. No me satisface dejar que las cosas sucedan y simplemente contemplarlas.

142. No me atrae conocer gente nueva.

143. Pocas veces sé cómo mantener una conversación.

144. Siempre tengo en cuenta los sentimientos de las personas.

145. Confío más en mis intuiciones que en mis observaciones.

146. Trato de no actuar hasta saber qué van a hacer los demás.

147. Me gusta tomar mis propias decisiones, evitando los consejos de los otros.

148. Muchas veces me siento muy mal sin saber por qué.

149. Me gusta ser popular y participar en muchas actividades sociales.

150. Raramente cuento a otro lo que pienso.

151. Me entusiasman casi todas las actividades que realizo.

152. Para mi es una práctica constante depender de mí mismo y no de otros.

153. La mayor parte del tiempo la dedico a organizar lo que tengo que hacer

154. No hay nada mejor que el afecto que se siente estando en medio del grupo familiar.

155. Algunas veces estoy tenso o deprimido sin saber por qué

156. Disfruto conversando sobre temas o sucesos místicos.

157. Decido cuales son las cosas prioritarias y luego actúo firmemente para poder lograrlas.

158. No dudo en orientar a las personas hacia lo que yo creo que es mejor para ellas.

159. Me siento orgulloso de ser eficiente y organizado. 
160. Realmente me desagradan las personas que se convierten en líderes sin razones que lo justifiquen.

161. Soy ambicioso en mis metas.

162. Sé cómo agradar a la gente

163. La gente puede confiar en que voy a hacer bien mi trabajo.

164. Los demás me consideran una persona más afectiva que racional.

165. Estaría dispuesto a trabajar mucho tiempo para poder llegar a ser alguien importante.

166. Me gustaría mucho poder vender nuevas ideas o productos a la gente

167. Generalmente logro convencer a los demás para que hagan exactamente lo que yo quiero.

168. Me gustan los trabajos en los que hay que prestar mucha atención en los detalles.

169. Soy muy introspectivo, siempre trato de entender mis pensamientos y emociones.

170. Confío mucho en mis habilidades sociales.

171. Evalúo las situaciones rápidamente y luego actúo para que las cosas salgan como yo quiero.

172. En una discusión soy capaz de convencer a casi todos para que apoyen mi posición.

173. Soy capaz de llevar a cabo cualquier trabajo, pese a los obstáculos que puedan presentarse.

174. Como si fuera un buen vendedor, puedo influir con éxito sobre los demás, con modales agradables.

175. Conocer gente nueva es un objetivo importante para mí.

176. Al tomar decisiones creo que lo más importante es pensar en el bienestar de la gente involucrada

177. Tengo paciencia para realizar trabajos que requieren mucha precisión.

178. Mi imaginación es superior a mi sentido de la realidad.

179. Estoy motivado para llegar a ser uno de los mejores en mi campo de trabajo.

180. Tengo un comportamiento que logra ganarme la aprobación de la gente. 


\section{Anexo B}

\section{Análisis de Confiabilidad - Consistencia Interna (alfa de Cronbach)}

\begin{tabular}{|c|c|c|c|c|c|c|}
\hline \multirow[b]{2}{*}{ Escalas del MIPS } & \multicolumn{3}{|c|}{ ltems Ponderados } & \multicolumn{3}{|c|}{ Items dicotómicos } \\
\hline & $\begin{array}{c}\text { Muestra } \\
\text { Total } \\
(n=533)\end{array}$ & $\begin{array}{l}\text { Adultos } \\
(n=143)\end{array}$ & $\begin{array}{c}\text { Estudiantes } \\
(n=390)\end{array}$ & $\begin{array}{c}\text { Muestra } \\
\text { Total } \\
(n=533)\end{array}$ & $\begin{array}{l}\text { Adultos } \\
(n=143)\end{array}$ & $\begin{array}{c}\text { Estudiantes } \\
(n=390)\end{array}$ \\
\hline 1A: Apertura & .7177 & .6490 & .7378 & .7294 & .6621 & .7493 \\
\hline 1B: Preservación & .7881 & .7424 & .8045 & .7828 & .7267 & .8030 \\
\hline 2A: Modificación & .7356 & .6227 & .7381 & .7399 & .6723 & .7335 \\
\hline 2B: Acomodación & .7413 & .6188 & .7611 & .7261 & 5968 & .7472 \\
\hline 3A: Individualismo & .6421 & .5786 & .6679 & .6124 & .5547 & 6357 \\
\hline 3B: Protección & .6680 & .6016 & .6648 & .6597 & .5730 & .6553 \\
\hline 4A: Extraversión & .7680 & .7208 & .7788 & .7761 & .7188 & .7906 \\
\hline 4B: Introversión & .7146 & .7014 & .7239 & .7348 & .7200 & .7427 \\
\hline 5A: Sensación & .6439 & .3971 & .6003 & .6558 & .3700 & .6092 \\
\hline 5B: Intuición & .6254 & .5537 & .5888 & .6342 & .5723 & .6049 \\
\hline 6A: Reflexión & .6641 & .5227 & .6900 & .6371 & .5295 & .6603 \\
\hline 6B: Afectividad & .6518 & .6094 & .6715 & .6319 & .6117 & .6476 \\
\hline $7 \mathrm{~A}:$ Sistematización & .8176 & .7942 & .7901 & .7794 & .7232 & .7586 \\
\hline 7B: Innovación & .6133 & .5395 & .6082 & .6501 & .5649 & .6352 \\
\hline 8A: Retraimiento & .7241 & .6922 & .7361 & .7407 & .6965 & .7545 \\
\hline 8B: Comunicatividad & .7776 & .7564 & .7798 & .7785 & .7616 & .7806 \\
\hline 9A: Vacilación & .8264 & .7794 & .8414 & .8285 & .7869 & .8412 \\
\hline 9B: Fimeza & .7882 & .7586 & .7902 & .7798 & .7229 & .7885 \\
\hline 10A: Discrepancia & .6592 & .5115 & .7020 & .7455 & .6579 & .7643 \\
\hline 10B: Conformismo & .7434 & .6385 & .7067 & .7793 & .7337 & .7338 \\
\hline 11A: Sometimiento & .6763 & .6473 & .6979 & .6476 & .6089 & .6712 \\
\hline 11B: Control & .6451 & .5879 & .6670 & .6404 & .5889 & .6644 \\
\hline 12A: Insatisfacción & .7133 & .6670 & .7315 & .7561 & 6391 & .7896 \\
\hline 12B: Concordancia & .6303 & .4973 & .6656 & .6329 & .4728 & .6783 \\
\hline
\end{tabular}


Anexo C

Validez Interna Estructural - Matriz de correlaciones entre las escalas

\begin{tabular}{|c|c|c|c|c|c|c|c|c|c|c|c|c|c|c|c|c|c|c|c|c|c|c|c|c|c|}
\hline & AlA & $A \mid B$ & $\mathrm{~A} 2 \mathrm{~A}$ & $\mathrm{~A} 2 \mathrm{~B}$ & $A B A$ & $\mathrm{~A} 3 \mathrm{~B}$ & $\mathrm{~A} 4 \mathrm{~A}$ & $\mathrm{~A} 4 \mathrm{~B}$ & $\mathrm{~A} 5 \mathrm{~A}$ & $\mathrm{~A} 5 \mathrm{~B}$ & $\mathrm{~A} 6 \mathrm{~A}$ & $A \in B$ & $\mathrm{~A} 7 \mathrm{~A}$ & $A>B$ & $A 8 \mathrm{~A}$ & $A 8 B$ & $\mathrm{~A} 9 \mathrm{~A}$ & $\mathrm{~A} 9 \mathrm{~B}$ & $\mathrm{AlOA}$ & $\mathrm{A} 10 \mathrm{~B}$ & AllA & $\mathrm{A} / 1 \mathrm{~B}$ & $\mathrm{~A} \mid 2 \mathrm{~A}$ & $A 12 B$ & AIP \\
\hline $\mathrm{A} I \mathrm{~B}$ & -851 & & & & & & & & & & & & & & & & & & & & & & & & \\
\hline $\mathrm{A} 2 \mathrm{~A}$ & .457 & -.313 & & & & & & & & & & & & & & & & & & & & & & & \\
\hline $\mathrm{A} 2 \mathrm{~B}$ & -.501 & .488 & -.777 & & & & & & & & & & & & & & & & & & & & & & \\
\hline$\overline{A 3 A}$ & .090 & .004 & .394 & -.201 & & & & & & & & & & & & & & & & & & & & & \\
\hline $\mathrm{A} 3 \mathrm{~B}$ & .195 & -.090 & .147 & -.045 & -.507 & & & & & & & & & & & & & & & & & & & & \\
\hline$\overline{\mathrm{A} 4 \mathrm{~A}}$ & .591 & -.535 & .545 & -.476 & .037 & .380 & & & & & & & & & & & & & & & & & & & \\
\hline $\mathrm{A} 4 \mathrm{~B}$ & -.532 & 6.30 & -.325 & .438 & .113 & -199 & .787 & & & & & & & & & & & & & & & & & & \\
\hline $\mathrm{A} 5 \mathrm{~A}$ & .175 & -181 & .257 & -.158 & .092 & .014 & .097 & \begin{tabular}{|c|}
-103 \\
\end{tabular} & & & & & & & & & & & & & & & & & \\
\hline $\mathrm{A} 5 \mathrm{~B}$ & -104 & .247 & -.026 & .137 & .155 & .089 & .053 & \begin{tabular}{|l|}
.173 \\
\end{tabular} & -.710 & & & & & & & & & & & & & & & & \\
\hline $\mathrm{A} 6 \mathrm{~A}$ & .042 & .070 & .342 & -.174 & 519 & -.369 & -116 & .217 & .286 & -.167 & & & & & & & & & & & & & & & \\
\hline $\mathrm{A} 6 \mathrm{~B}$ & .068 & .190 & -.045 & .202 & -321 & .705 & 214 & -.029 & -.274 & .483 & -.577 & & & & & & & & & & & & & & \\
\hline A7A & .358 & -293 & .745 & -.683 & .134 & .155 & .327 & -.281 & .385 & -263 & 295 & -103 & & & & & & & & & & & & & \\
\hline A7B & .013 & $.12 I$ & .007 & 148 & .279 & .017 & .270 & \begin{tabular}{|l|}
.018 \\
\end{tabular} & -.421 & .648 & -138 & .378 & -.463 & & & & & & & & & & & & \\
\hline$\overline{A 8 A}$ & -.584 & .591 & -.412 & .471 & .227 & -.436 & -.792 & .781 & -132 & .140 & .302 & -194 & -.342 & .013 & & & & & & & & & & & \\
\hline $\mathrm{A} 8 \mathrm{~B}$ & .600 & -.466 & .742 & -.581 & .240 & .294 & .794 & -.557 & .146 & .094 & .144 & .143 & .494 & .205 & -.672 & & & & & & & & & & \\
\hline A9A & -815 & .811 & -.464 & .566 & -082 & -.152 & -.716 & \begin{tabular}{|l|}
.738 \\
\end{tabular} & -.174 & 139 & -.003 & .081 & -384 & .011 & .666 & -.670 & & & & & & & & & \\
\hline $\mathrm{A} 9 \mathrm{~B}$ & .692 & -.570 & .696 & .697 & .340 & .100 & .580 & -.488 & .177 & -.045 & \begin{tabular}{r|}
.284 \\
\end{tabular} & \begin{tabular}{l|l|}
-.088 \\
-10
\end{tabular} & .584 & \begin{tabular}{l|l|} 
& 029 \\
\end{tabular} & -489 & \begin{tabular}{|l|}
.758 \\
\end{tabular} & -.784 & & & & & & & & \\
\hline $\mathrm{AlOA}$ & -.442 & .497 & .211 & 431 & .514 & -.411 & -264 & \begin{tabular}{|l|}
.417 \\
\end{tabular} & -.087 & .304 & .220 & $\begin{array}{l}-.083 \\
\end{array}$ & $\begin{array}{r}-419 \\
\end{array}$ & .499 & .539 & -246 & .447 & -308 & & & & & & & \\
\hline $\mathrm{AlOB}$ & .385 & -.272 & .626 & -.471 & .051 & .336 & .326 & -233 & .541 & -.337 & .305 & .026 & .751 & -.443 & -362 & .510 & -.37 .3 & .534 & -.460 & & & & & & \\
\hline AllA & -699 & .683 & -.459 & 603 & .148 & .006 & -430 & .494 & -157 & .232 & -.087 & .204 & .432 & .156 & \begin{tabular}{l|l|}
492 \\
\end{tabular} & -.471 & .728 & -698 & \begin{tabular}{|l|}
533 \\
\end{tabular} & -344 & & & & & \\
\hline AllB & .235 & -.114 & .494 & -.320 & .695 & .260 & .247 & .073 & .092 & .144 & $\begin{array}{l}.488 \\
\end{array}$ & .220 & .221 & .307 & .007 & .448 & -.281 & .563 & .325 & .134 & -328 & & & & \\
\hline $\mathrm{A} 12 \mathrm{~A}$ & -.612 & .676 & -201 & .443 & .311 & -.348 & -.367 & .527 & -121 & .262 & .268 & -.060 & -297 & .294 & .591 & -.280 & .595 & -396 & .742 & -309 & .600 & .189 & & & \\
\hline $\mathrm{A} 12 \mathrm{~B}$ & -.015 & .025 & -.271 & .254 & -.712 & .676 & .011 & -064 & -.042 & -048 & -495 & .540 & -013 & -252 & -.178 & -.180 & .098 & -295 & -.441 & \begin{tabular}{|l|}
148 \\
\end{tabular} & .176 & -704 & \begin{tabular}{|c|}
351 \\
\end{tabular} & & \\
\hline AIP & .186 & -.071 & .327 & -.241 & .005 & .241 & .070 & -.025 & .257 & -143 & .286 & .013 & .417 & -296 & -.087 & .185 & -100 & .248 & -261 & .541 & -.174 & \begin{tabular}{|l|}
.018 \\
\end{tabular} & -0.178 & $\mid .134$ & \\
\hline AIN & -.695 & .829 & -284 & .544 & .063 & -.062 & -.442 & .607 & -.147 & .271 & .118 & .186 & -330 & .221 & .599 & -.373 & .755 & -.541 & .593 & -.250 & .684 & .075 & 722 & -.002 & $\cdot 00$ \\
\hline
\end{tabular}




\section{Anexo D}

\section{Comparación de la muestra universitaria peruana con la norteamericana}

\begin{tabular}{|c|c|c|c|c|c|c|}
\hline \multirow[t]{2}{*}{ Escalas del MIPS } & \multicolumn{2}{|c|}{$\begin{array}{l}\text { PERÚ } \\
(390)\end{array}$} & \multicolumn{2}{|c|}{$\begin{array}{l}\text { EEUU } \\
(1000)\end{array}$} & \multirow[b]{2}{*}{$t$} & \multirow[b]{2}{*}{$d$} \\
\hline & Media & $D E$ & Media & $D E$ & & \\
\hline IA: Apertura & 24.14 & 76 & 24.8 & 8.7 & -1.692 & 0.079 \\
\hline IB: Preservación & 17.99 & 10.5 & 16.8 & 11.8 & $2.245^{*}$ & 0.104 \\
\hline 2A: Modificación & 29.32 & 8.5 & 29.2 & 10.8 & 0.285 & 0.012 \\
\hline 2B: Acomodación & 19.72 & 9.8 & 19.0 & 11.8 & 1.448 & 0.064 \\
\hline 3A: Individualismo & 22.10 & 7.5 & 19.9 & 8.6 & $5.756^{* * *}$ & 0.265 \\
\hline 3B: Protección & 27.88 & 7.9 & 29.6 & 9.2 & $-4.275^{* * *}$ & 0.194 \\
\hline 4A: Extraversión & 25.75 & 8.8 & 28.6 & 9.3 & $-6.315^{* * *}$ & 0.311 \\
\hline 4B: Introversión & 12.57 & 7.3 & 11.4 & 7.7 & $3.139^{* *}$ & 0.154 \\
\hline 5A: Sensación & 15.28 & 6.1 & 14.5 & 7.2 & $2.543^{*}$ & 0.113 \\
\hline 5B: Intuición & 25.65 & 7.4 & 26.3 & 8.7 & -1.707 & 0.078 \\
\hline 6A: Reflexión & 21.63 & 8.1 & 17.1 & 9.2 & $10.950 * * *$ & 0.509 \\
\hline 6B: Afectividad & 27.72 & 7.8 & 29.8 & 9.3 & $-5.217^{* * *}$ & 0.234 \\
\hline 7A: Sistematización & 33.92 & 11.0 & 33.7 & 11.9 & 0.410 & 0.019 \\
\hline 7B: Innovación & 31.01 & 8.0 & 31.7 & 9.6 & -1.689 & 0.075 \\
\hline 8A: Retraimiento & 19.45 & 9.5 & 16.8 & 10.2 & $5.518 * * *$ & 0.265 \\
\hline 8B: Comunicatividad & 35.74 & 10.4 & 35.6 & 11.7 & 0.276 & 0.012 \\
\hline 9A: Vacilación & 18.22 & 11.4 & 16.8 & 12.2 & $2.463^{*}$ & 0.119 \\
\hline 9B: Firmeza & 37.20 & 10.0 & 33.9 & 11.3 & $6.520 * * *$ & 0.301 \\
\hline 10A: Discrepancia & 22.67 & 8.9 & 22.4 & 9.7 & 0.612 & 0.028 \\
\hline 10B: Conformismo & 39.24 & 8.8 & 36.6 & 10.5 & $5.899^{* * * *}$ & 0.263 \\
\hline 11A: Sometimiento & 15.50 & 7.9 & 18.9 & 9.2 & $-8.402 * * *$ & 0.384 \\
\hline 11B: Control & 26.54 & 7.6 & 21.0 & 9.1 & $14.312 * * *$ & 0.636 \\
\hline 12A: Insatisfacción & 25.38 & 10.2 & 24.0 & 10.7 & $2.676^{* *}$ & 0.131 \\
\hline 12B: Concordancia & 30.93 & 8.5 & 33 & 10.8 & $-4.779^{* * *}$ & 0.203 \\
\hline
\end{tabular}

${ }^{* * *} p<.001,{ }^{* *} p<.01,{ }^{*} p<.05$ 
Adaptación del MIPS

Anexo E

Comparación de la muestra universitaria peruana con la española

\begin{tabular}{|l|c|c|c|c|c|c|}
\hline \multirow{2}{*}{ Escalas del MIPS } & \multicolumn{2}{|c|}{$\begin{array}{c}\text { PERÚ } \\
(390)\end{array}$} & \multicolumn{2}{c|}{$\begin{array}{c}\text { ESPANA } \\
(1000)\end{array}$} & \multicolumn{1}{c|}{} \\
\cline { 2 - 7 } & Media & $D E$ & Media & $D E$ & $t$ & $d$ \\
\hline 1A: Apertura & 24.14 & 7.6 & 22.87 & 7.6 & $3.322^{* * *}$ & 0.167 \\
\hline 1B: Preservación & 17.99 & 10.5 & 18.62 & 10.5 & -1.167 & 0.060 \\
\hline 2A: Modificación & 29.32 & 8.5 & 25.98 & 8.9 & $7.696^{* * *}$ & 0.380 \\
\hline 2B: Acomodación & 19.72 & 9.8 & 21.90 & 9.8 & $-4.380^{* * *}$ & 0.222 \\
\hline 3A: Individualismo & 22.10 & 7.5 & 18.73 & 8.1 & $8.806^{* * *}$ & 0.425 \\
\hline 3B: Protección & 27.88 & 7.9 & 29.00 & 7.9 & $-2.779^{* *}$ & 0.142 \\
\hline \multicolumn{7}{|c|}{} \\
\hline 4A: Extraversión & 25.75 & 8.8 & 26.56 & 8.7 & -1.786 & 0.093 \\
\hline 4B: Introversión & 12.57 & 7.3 & 11.15 & 6.7 & $3.808^{* * *}$ & 0.207 \\
\hline 5A: Sensación & 15.28 & 6.1 & 15.49 & 6.6 & -0.655 & 0.032 \\
\hline 5B: Intuición & 25.65 & 7.4 & 23.47 & 8.3 & $5.799^{* * *}$ & 0.271 \\
\hline 6A: Reflexión & 21.63 & 8.1 & 16.76 & 8.5 & $11.772^{* * *}$ & 0.580 \\
\hline 6B: Afectividad & 27.72 & 7.8 & 28.84 & 8.6 & $-2.806^{* *}$ & 0.134 \\
\hline 7A: Sistematización & 33.92 & 11.0 & 31.88 & 11.46 & $3.677^{* * *}$ & 0.180 \\
\hline 7B: Innovación & 31.01 & 8.0 & 27.29 & 9.0 & $9.111^{* * *}$ & 0.426 \\
\hline & \multicolumn{7}{|c|}{} & & & \\
\hline 8A: Retraimiento & 19.45 & 9.5 & 16.99 & 8.8 & $5.124^{* * *}$ & 0.273 \\
\hline 8B: Comunicatividad & 35.74 & 10.4 & 32.63 & 10.0 & $5.886^{* * *}$ & 0.308 \\
\hline 9A: Vacilación & 18.22 & 11.4 & 18.45 & 10.7 & -0.393 & 0.021 \\
\hline 9B: Firmeza & 37.20 & 10.0 & 30.94 & 10.6 & $12.360^{* * *}$ & 0.600 \\
\hline 10A: Discrepancia & 22.67 & 8.9 & 22.24 & 8.9 & 0.965 & 0.048 \\
\hline 10B: Conformismo & 39.24 & 8.8 & 34.13 & 9.3 & $11.416^{* * *}$ & 0.558 \\
\hline 11A: Sometimiento & 15.50 & 7.9 & 17.00 & 8.0 & $-3.707^{* * *}$ & 0.188 \\
\hline 11B: Control & 26.54 & 7.6 & 22.12 & 7.55 & $11.423^{* * *}$ & 0.584 \\
\hline 12A: Insatisfacción & 25.38 & 10.2 & 23.82 & 9.5 & $3.024^{* *}$ & 0.161 \\
\hline 12B: Concordancia & 30.93 & 8.5 & 35.21 & 9.3 & $-9.890^{* * *}$ & 0.471 \\
\hline
\end{tabular}

${ }^{* * *} p<.001,{ }^{* *} p<.01,{ }^{*} p<.05$ 